$\underline{\text { Report }}$

\title{
Examination of the Taxonomic Position of Penicillium Strains Used in Blue Cheese Production Based on the Partial Sequence of $\beta$-Tubulin
}

(Received December 5, 2013)

\author{
Yoshio Ogawa ${ }^{1, *}$, Dai Hirose ${ }^{1}$, Ayano AkiYama ${ }^{1}$ and Masakatsu Ichinoe ${ }^{2,3}$ \\ ${ }^{1}$ School of Pharmacy, Nihon University: 7-7-1 Narashinodai, \\ Funabashi, Chiba 274-8555, Japan; \\ ${ }^{2}$ Tokyo Kasei University: 1-18-1 Kaga, Itabashi, Tokyo \\ 173-0033, Japan; \\ ${ }^{3}$ Mycotoxin Research Association: 15 Daikoku Futo, \\ Tsurumi, Yokohama 230-0054, Japan; \\ * Corresponding author
}

\begin{abstract}
Penicillium roqueforti is a well known starter used for blue cheese production. Two closely related species, $P$. carneum and $P$. paneum, were previously classified as varieties of $P$. roqueforti. Penicillium roqueforti does not produce patulin, a mycotoxin harmful for human health, whereas both $P$. carneum and $P$. paneum actively produce this toxin. From the viewpoint of food safety, it is thus important to confirm that $P$. carneum and $P$. paneum are not used for cheese production. In the present study, the taxonomic position of Penicillium strains used for blue cheese production was examined on the basis of the partial sequence of $\beta$-tubulin. Twenty-eight Penicillium strains isolated from blue cheeses were investigated. All the examined strains belonged to P. roqueforti. Therefore, the Penicillium strains used for production of the blue cheese samples examined here do not negatively impact on human health.
\end{abstract}

Key words: blue cheese; $\beta$-tubulin; Penicillium roqueforti; taxonomic position

\section{Introduction}

Penicillium roqueforti Thom is the fungus used for blue cheese production. In the production of blue cheeses, strains of this fungus are inoculated into cow, sheep, or goat milk so that final products are spotted or veined throughout with blue, blue-gray, or blue-green mold, and exhibit characteristic flavors.

Typical strains of $P$. roqueforti are characterized by dark-green to blackish-green reverse ${ }^{1)}$. However, Frisvad and Filtenborg discovered that some strains of this species show pale-brown colony reverse, and these strains differ from typical strains in having a sweet, moldy smell, lower average growth rate, and mycotoxin production $^{2}$. They thus distinguished such strains as a separate variety, $P$. roqueforti var. carneum Frisvad, from $P$. roqueforti Thom var. roqueforti. Furthermore, Boysen et al. split $P$. roqueforti var. carneum into two species based on sequence analysis of nuclear rDNA internal transcribed spacer (ITS) region, including 5.8S rDNA, random amplified polymorphic DNA (RAPD) genotype, and mycotoxin production ${ }^{3)}$. At present, therefore, the $P$. roqueforti group has been reclassified into

\footnotetext{
* ogawa.yoshio@nihon-u.ac.jp
}

the following three species: $P$. roquefort $i$ Thom, $P$. carneum Frisvad, and $P$. paneum Frisvad.

These species of the $P$. roqueforti group produce several kinds of mycotoxins. Roquefortine $\mathrm{C}$ and mycophenolic acid are produced by $P$. roqueforti and $P$. carneum $^{3)}$, and exhibit very low toxicities ${ }^{4)}$. PR toxin, which is produced only by $P$. roqueforti $i^{3)}$, inhibits in vivo and/or in vitro protein synthesis and nucleic acid synthesis ${ }^{5), 6)}$. Furthermore, several researchers have reported that PR toxin is both cytotoxic ${ }^{6), 7)}$ and mutagenic ${ }^{8)}$. However, as $\mathrm{PR}$ toxin is not stable in cheese and breaks down into the less toxic PR imine ${ }^{9), 10)}$, the human health hazard is very low. Patulin is produced by $P$. carneum and $P$. paneum $^{3)}$. Many investigators have emphasized the toxicities of patulin such as carcinogenicity ${ }^{11)}$, cytotoxicity $^{12)}$, clastogenicity ${ }^{13)}$, mutagenicity ${ }^{14)}$, immunotoxicity ${ }^{15)}$ and impact on intestinal function, ${ }^{16), 17)}$ although some reports have questioned its carcinogenicity and immunotoxicity ${ }^{18)}$ 19). Therefore, intake of patulin from contaminated food should be kept to a minimum.

From the viewpoint of food safety, it is important to confirm that the fungi used for blue cheese production are $P$. roqueforti strains that do not produce patulin. Frisvad and Filtenborg ${ }^{2}$ found that the Penicillium strains isolated from blue cheeses were solely $P$. 
roqueforti, and stated that $P$. carneum and $P$. paneum were never found in blue cheeses. However, data to support this statement were not presented in their paper. Shimada and Ichinoe isolated Penicillium strains from various blue cheeses produced in Europe and $\operatorname{Japan}^{20), 21)}$. In the present study, we analyzed the partial sequences of $\beta$-tubulin of these Penicillium strains and examined the taxonomic position of these strains among $P$. roqueforti and related species.

\section{Materials and Methods}

Among the Penicillium strains isolated by Shimada and Ichinoe ${ }^{20)}$ 21), 28 strains were used for the present study (Table 1). To clarify the taxonomic position of these Penicillium strains within the $P$. roqueforti group, partial sequences of $\beta$-tubulin were determined.

DNA was extracted from the mycelia of each fungal strain, cultured at room temperature in $2.5 \%$ malt extract broth (Bacto ${ }^{\mathrm{TM}}$ Malt Extract, Becton, Dickinson and Company, Sparks, MD), using a DNeasy Plant Mini Kit (Qiagen, Hilden, Germany). The partial $\beta$-tubulin sequences were amplified by PCR with primers TUB638L21 (ccttagcceagttgttaccag: Iwamoto unpublished) and TUB119U18 (accggccagtgtgtaagt; Iwamoto unpublished). PCR was performed using HotStarTaq Plus Master Mix (Qiagen) under the following conditions: initial incubation of $95^{\circ} \mathrm{C}$ for $5 \mathrm{~min}, 35$ cycles of $95^{\circ} \mathrm{C}$ for $30 \mathrm{~s}, 54^{\circ} \mathrm{C}$ for $30 \mathrm{~s}$, and $72^{\circ} \mathrm{C}$ for $90 \mathrm{~s}$, and a final extension period of $72^{\circ} \mathrm{C}$ for $10 \mathrm{~min}$. The PCR products were purified with a QiAquick PCR Purification Kit (Qiagen) and sequenced by Macrogen Japan Inc. (Tokyo, Japan). The obtained sequences consisted of 420421 bps including 183 bps of intron.

Several sequences were retrieved from the nucleotide database of NCBI for comparison with the sequences determined in the present study. The sources are listed in Table 2. DNA sequences were aligned through a profile/structure alignment process in CLUSTAL W ver. $1.71^{22)}$. Finally, the aligned data were checked and optimized manually.

The phylogenetic relationships of $P$. roqueforti strains and related species were analyzed by the neighbour-

Table 1. Penicillium roqueforti strains used in the present study, and their morphological type $\mathrm{e}^{\mathrm{a})}$ and mycotoxin-producing ability

\begin{tabular}{|c|c|c|c|c|c|c|c|c|}
\hline \multirow{3}{*}{ Strain } & \multirow{3}{*}{ Cheese as substratum } & \multirow{3}{*}{ Origin } & \multirow{3}{*}{$\begin{array}{l}\text { Morphological } \\
\text { type }\end{array}$} & \multicolumn{4}{|c|}{ Mycotoxin-producing ability } & \multirow{3}{*}{ Acc. No. } \\
\hline & & & & \multicolumn{2}{|c|}{ Mycophenolic acid } & \multicolumn{2}{|c|}{ Roquefortin $\mathrm{C}$} & \\
\hline & & & & $14^{\circ} \mathrm{C}$ & $16^{\circ} \mathrm{C}$ & $14^{\circ} \mathrm{C}$ & $16^{\circ} \mathrm{C}$ & \\
\hline $96-\mathrm{B}$ & Roquefort & France & IV & + & + & + & + & AB813843 \\
\hline 96-D & Roquefort & France & IV & + & + & + & + & AB813844 \\
\hline $96-\mathrm{N}$ & Roquefort & France & IV & + & + & + & + & AB813845 \\
\hline $97-\mathrm{O}^{\mathrm{c})}$ & Roquefort & France & & + & + & + & + & AB813846 \\
\hline $97-B^{c)}$ & Fourme d'Ambert & France & & & & & & AB813847 \\
\hline $98-B^{c)}$ & Fourme d'Ambert & France & & & & & & AB813848 \\
\hline $98-\mathrm{F}^{\mathrm{c})}$ & Lys bleu le Bleu du Velay & France & & - & - & + & + & AB813849 \\
\hline $99-A^{c)}$ & Forez Fourme & France & & & & & & AB813850 \\
\hline 96-Ra & Gorgonzola & Itali & I & - & - & + & + & AB813851 \\
\hline $96-\mathrm{Wa}$ & Gorgonzola & Itali & I & - & - & + & + & AB813852 \\
\hline $96-\mathrm{Sa}^{\mathrm{c}), \mathrm{d})}$ & Stilton & England & & - & - & + & + & AB813853 \\
\hline $96-\mathrm{Sb}^{\mathrm{d})}$ & Stilton & England & III & - & - & + & + & AB813854 \\
\hline $96-\mathrm{Ea}^{\mathrm{c}), \mathrm{e}}$ & Danish blue & Denmark & & + & + & + & + & AB813855 \\
\hline $96-\mathrm{Eb}^{\mathrm{e})}$ & Danish blue & Denmark & II & - & - & + & + & AB813856 \\
\hline $96-\mathrm{V}$ & Danish blue & Denmark & II & - & - & + & + & AB813857 \\
\hline $96-\mathrm{G}$ & Castello blue & Denmark & II & - & - & + & + & AB813858 \\
\hline $98-L^{c)}$ & Crème Royale & Denmark & & & & & & AB813859 \\
\hline $96-\mathrm{Fa}$ & Blue Brie (Cambozola) & Germany & I & - & - & + & + & AB813860 \\
\hline $96-\mathrm{Ua}$ & Blue Brie (Babaria blue) & Germany & III & - & - & + & + & AB813861 \\
\hline $99-B^{c)}$ & Gippsland Blue & Austria & & & & & & AB813862 \\
\hline $97-\mathrm{E}^{\mathrm{c})}$ & Normanna & Norway & & & & & & AB813863 \\
\hline $98-\mathrm{Da}^{\mathrm{c}}$ & Normanna & Norway & & & & & & AB813864 \\
\hline 99- $\mathrm{Da}^{\mathrm{c}}$ & Normanna & Norway & & & & & & AB813865 \\
\hline $96-C^{c)}$ & Blue-K (Kobuchizawa) & Japan & & + & + & + & + & AB813866 \\
\hline $96-\mathrm{H}^{\mathrm{c})}$ & Blue-S (Shosenkyo) & Japan & & & & & & AB813867 \\
\hline $96-\mathrm{I}$ & Blue veined camembert & Japan & $\mathrm{V}$ & + & + & + & + & AB813868 \\
\hline $96-\mathrm{K}$ & Blue-H (Hokkaido) & Japan & $\mathrm{V}$ & + & + & + & + & AB813869 \\
\hline 97-Ca & Kobuchizawa camembert and blue & Japan & IV & + & + & + & + & AB813870 \\
\hline
\end{tabular}

a) The data of morphological types were retrieved from Shimada and Ichionoe ${ }^{20)}$.

b) The data of mycotoxin productivities were retrieved from Shimada and Ichinoe ${ }^{21)}$. In the original paper, mycophenolic acid production was shown quantitatively. In the present study, it is shown qualitatively.

c) The morphological types and/or mycotoxin productivities were not studied.

d) Strains 96-Sa and 96-Sb were isolated from the same Stilton cheese.

e) Strains 96-Ea and 96-Eb were isolated from the same Danish blue cheese. 
Table 2. $\quad \beta$-Tubulin sequences of Penicillium isolates obtained from various databases (DDBJ/EMBL/GenBank)

\begin{tabular}{llll}
\hline \hline \multicolumn{1}{c}{ Strain } & Taxon name & Source & Acc. No. \\
CBS 484.84 & P. aethiopicum & Grain of Hordeum vulgare & AY495983 \\
CBS 449.78 & P. carneum & Cheddar cheese & AY674384 \\
CBS 112297 & P. carneum & Mouldy rye bread & AY674386 \\
CNU 7003 & P. expansum & Apple & HQ225727 \\
OY127E & P. paneum & Grass silage & EU090072 \\
OY112C & P. paneum & Grass silage & EU090085 \\
CBS 135.67 & P. roqueforti & Blue cheese & AY674380 \\
CBS 479.84 & P. roqueforti & Mouldy bakers yeast & AY674382 \\
CBS 221.30 & P. roqueforti & French roqueforti cheese & AY674383 \\
TN115K & P. roqueforti & Grass silage & EU090086 \\
LM210A & P. roqueforti & Grass silage & EU090100 \\
KY219A & P. roqueforti & Grass silage & EU090087 \\
\hline
\end{tabular}

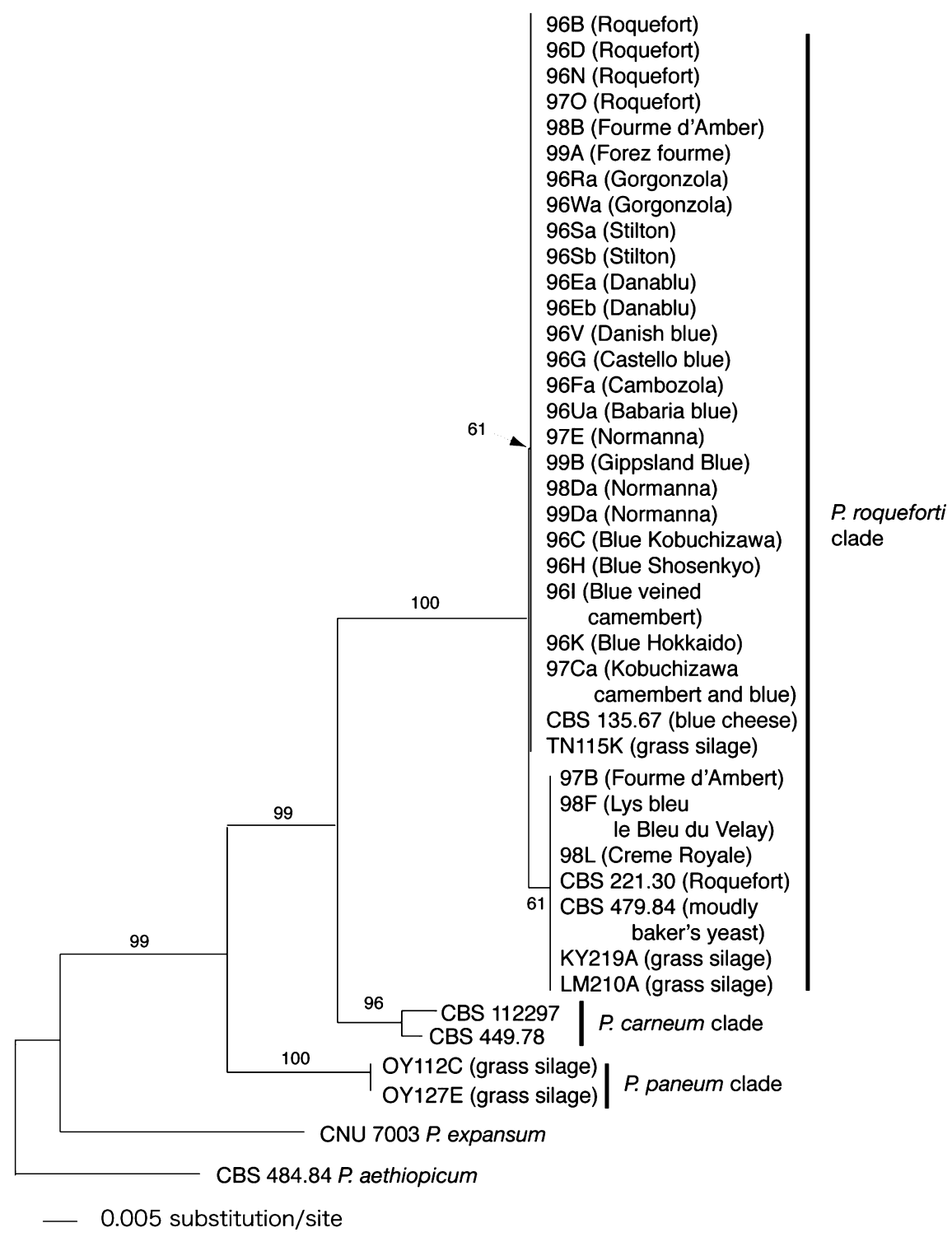

Fig. 1. The relationship between Penicillium roqueforti, P. carneum and P. paneum, inferred from Neighbour-Joining analysis of the partial sequences of $\beta$-tubulin.

Percentages beside the branches are bootstrap values of 1,000 replicates. The substrates from which strains were isolated are shown in parentheses. When the substrate was cheese, the name of the cheese is shown in most cases. 
joining (NJ) method ${ }^{23)}$, using PAUP* version 4.0 beta 10 $(4.0 \mathrm{~b} 10)^{24)}$. The examined operational taxonomic units provided 430 aligned sites including sequence gaps. All indels were excluded from the phylogenetic analysis. Penicillium aethiopicum was selected as the outgroup. The distances between OTUs were estimated by the Hasegawa, Kishino, and Yano (HKY85) model ${ }^{25)}$ without the assumption of rate heterogeneity. Stability of clades was assessed by 1,000 bootstrap replications.

\section{Results and Discussion}

As a result of NJ analysis, the 28 Penicillium strains isolated by Shimada and Ichinoe were resolved into the $P$. roqueforti clade with other $P$. roqueforti strains listed in Table 2, and this clade was supported by a high bootstrap value of $100 \%$ (Fig. 1). The two strains of $P$. carneum, CBS 449.78 and CBS112297, and the two P. paneum strains, OY112C and OY127E, created separate clades (Fig. 1). Penicillium carneum clade and P. paneum clade were also supported by high bootstrap values, $96 \%$ and $100 \%$, respectively.

These results clearly demonstrated that only $P$. roqueforti was used for production of the blue cheese samples examined here, and that the two closely related species, and $P$. carneum and $P$. paneum, were not employed. Therefore, the toxicological risk of patulin intake by consumption of blue cheeses appears to be minimal.

O'Brien et al. investigated the molecular characteristics of 38 isolates of $P$. roqueforti from baled grass silage $^{26)}$. They showed that the partial sequences of $\beta$ tubulin resolved these isolates into three groups. In the present study, representative strains of each group, TNK115K, LM210A and KY219A, were analyzed together with the blue cheese strains. All three strains fell into the $P$. roqueforti clade with the strains isolated from blue cheeses (Fig. 1). Judging from the partial sequences of $\beta$-tubulin, we surmise that $P$. roqueforti strains are not specialized for blue cheese production, and that they do not differ from strains dwelling in natural habitats.

Shimada and Ichinoe ${ }^{20)}$ studied the morphological characteristics of $P$. roqueforti isolates from blue cheeses and divided the strains into five types based on the color of colony reverse, sclerotium formation and conidiophore ornamentation. They determined the morphological types of 14 of the 28 strains used in the present study (Table 1). We could not find a clear relationship between the morphological types and the topology of the NJ tree (Fig. 1). In spite of the morphological types, all strains were resolved together into the $P$. roqueforti clade.

Shimada and Ichnoe ${ }^{21)}$ also investigated mycotoxin production of $P$. roqueforti strains. Among the 28 strains used for the present study, 20 strains produce mycotoxins (Table 1). Nineteen strains produced roquefortin C whereas only 9 strains produced mycophenolic acid. The topology of the NJ tree (Fig. 1) did not reflect the difference in mycotoxin production ability of these strains as a difference in morphological type.

The NJ dendrogram based on the partial $\beta$-tubulin sequence did not show clades correspond to intraspecific variations in morphology and mycotoxin production in $P$. roqueforti. In order to detect these intraspecific variations, we need to use more sensitive markers such as RAPD and AFLP (amplified fragment length polymorphism).

\section{References}

1) Ramírez, C. Manual and Atlas of the Penicillia. Amsterdam: Elsevier, 1982, p. 381-385. (ISBN: 0-444-803696)

2) Frisvad, J. C., Filtenborg, O. Terverticillate penicillia: chemotaxonomy and mycotoxin production. Micologia, 81, 837-861 (1989).

3) Boysen, M., Skouboe, P., Frisvad, J., Rossen, L. Reclassification of the Penicillium roqueforti group into three species on the basis of molecular genetic and biochemical profiles. Microbiology, 142, 541-549 (1996).

4) Scott, P. M. Toxins of Penicillium species used in cheese manufacture. J. Food Prot., 44, 702-710 (1981).

5) Moulè, Y., Jemmali, M., Darracq. N. Inhibition of protein synthesis by PR toxin, a mycotoxin from Penicillium roqueforti. FEBS Lett., 88, 341-344 (1978).

6) Aujard, C., Morel-Chany, E., Icard, C., Trical. G. Effects of PR toxin on liver cells in culture. Toxicology., 12 313-323 (1979).

7) Lompe, A., von Milczewski. K. E. Ein Zellkulturtest für den Nachweis von Mykotoxinen. I. Untersuchungen an Reinsubustanzen. Z. Lebesm. Unters.-Forsch., 169, 249254 (1979)

8) Ueno, Y., Kubota, K., Ito, T., Nakamura, Y. Mutagenicity of carcinogenic mycotoxins in Salmonella Typhimurium. Cancer Res., 38, 536-542 (1978).

9) Chang, S., Lu, K., Yeh, S. Secondary metabolites resulting from degradation of PR-toxin by Penicillium roqueforti. Appl. Environ. Microbiol., 59, 981-986 (1993)

10) Engel, G., Orokopek, D. Kein Nachweis von Penicillium roqueforti-Käse, Milchwissenschaft, 34, 272-274 (1979).

11) Dickens, F., Jones, H. E. H. Carcinogenic activity of a series of reactive lactones and related substances. Br. J. Cancer, 15, 85-100 (1961).

12) Barhoumi, R., Burghardt, R. C. Kinetic analysis of the chronology of patulin- and gossypol-induced cytotoxicity in vitro. Toxicol. Sci., 30, 290-297 (1996).

13) Pfeiffer, E., Groß, K., Metzler, M. Aneuploidogenic and clastogenic potential of the mycotoxins citrinin and patulin. Carcinogenesis, 19, 1313-1318 (1998).

14) Roll, R., Matthiaschk, G., Korte, A. Embryotoxicity and mutagenicity of mycotoxins. J. Eniviron. Pathol. Toxicol. Oncol., 10, 1-7 (1990).

15) Escoul, L., Thomsen, M., Bourdiol, D., Pipy, B., Peuriere, S., Roubinet, F. Patulin immunotoxicology: Effect on phagocyte activation and the cellular and humoral immune system of mice and rabbits. Int. J. Immunopharmacol., 10, 983-989 (1988).

16) McKinley, E. R., Carlton, W. E. Patulin mycotoxicosis in Syrian hamster. Food Cosmet. Toxicol., 18, 173-179 (1980).

17) McKinley, E. R., Calton, W. W. Patulin mycotoxicosis in Swiss ICR mice. Food Cosmet. Toxicol., 18, 181-187 (1980)

18) Osswald, H., Frank, H. K., Komitowski, D., Winter, H. Long-term testing of patulin administered orally to Sprague-Dawley rats and Swiss mice. Food Cosmet. Toxicol., 16, 243-247 (1978)

19) Llewellyn, G. C., McCay, J. A., Brown, R. D., Musgrove, 
D. L., Butterworth, L. F., Munson, A. E., White, Jr. K. L. Immunological evaluation of the mycotoxin patulin in female B6C3F mice. Food and Chem. Toxicol., 36, 11071115 (1998)

20) Shimada, T., Ichinoe, M. Fungal species from imported and domestic mold-ripened cheese. Shokuhin Eiseigaku Zasshi (J. Food Hyg. Soc. Japan), 39, 350-356 (1998)

21) Shimada, T., Ichinoe, M. Production of toxic metabolites by Penicillium strains isolated from mold-ripened cheese. Shokuhin Eiseigaku Zasshi (Food Hyg. Saf. Sci.), 41, 126-132 (2000)

22) Thompson, J. D., Gibson, F., Plewniak, F., Jeanmougin, F., Higgins, D. G. The Clustal X-Windows interface: flexible strategies for multiple sequence alignment aided by quality analysis tools. Nucleic Acids Res., 25, 4876-
4882 (1997).

23) Saitou, N., Nei, M. The Neighbour-Joining method: a new method for reconstructing phylogenetic trees. Mol. Biol. Evol., 4, 406-425 (1987).

24) Swofford, D. L. PAUP*: phylogenetic analysis using parsimony and other methods (PAUP* version 4.0 beta 10). Sinauer, Sunderland (2001).

25) Hasegawa, M., Kishino, H., Yano, T. Dating of the human-ape splitting by a molecular clock of mitochondria DNA. J. Mol. Evol., 22, 160-174 (1985).

26) O’Brien, M. Egan, D., O’Kiely, P., Forristal, P. D., Doohan, F. M., Fuller, H. T. Morphological and molecular characterization Penicillium roqueforti and P. paneum isolated from baled grass silage. Mycol. Res., 112, 921932 (2008). 